\title{
Study Day of Macroscopic Findings
}

National Cancer Institute

\section{Source}

National Cancer Institute. Study Day of Macroscopic Findings. NCI Thesaurus. Code C119858.

The study day that a macroscopic findings assessment is performed. 